\title{
IT BUSINESS VALUE INDEX
}

\author{
Managing IT Investments for Business Value
}

\author{
Malvina Nisman \\ Intel Corporation*
}

\begin{abstract}
Traditional financial and accounting measures often fail to properly quantify the business benefits of IT investments. Intel's Business Value Index (BVI) methodology helps us prioritize our IT investments through a common language and framework for assessing potential business value, impact on IT efficiency, and financial attractiveness.
\end{abstract}

Key words: Intel Information Technology White Paper, IT investments, business value

\section{INTRODUCTION}

IT investments are growing more complex, more strategic, and larger in scope. At the same time, traditional financial and accounting measures often fail to properly quantify the business benefits of those investments. Intel IT strives to invest in projects that bring both business value and IT efficiency, and are also financially attractive. To prove such bottom-line results, we developed the IT Business Value (ITBV) program at the end of 2001 to forecast the real value of an IT project and document the actual business value it delivers to Intel.

The Business Value Index (BVI) methodology helps to prioritize investment options, make data-driven decisions, and monitor progress. It encompasses business value, IT efficiency value, and financial criteria. Business value measures the impact of a project on our business strategy and priorities. IT efficiency value gauges how well the investment will use or enhance our existing infrastructure. The financial criterion measures the financial attractiveness of the investment, including time to return investment, cost/benefit ratio, and net present value (NPV) of a project.

*An earlier version of this paper was published by Intel on IT@Intel. 
BVI complements analysis tools that measure return on investment (ROI). Using the BVI methodology, Intel's decision makers evaluate proposed IT investments based on potential business value, impact on IT efficiency, and financial attractiveness. Our decision makers also value early stage investments-managed as IT strategic options—using BVI.

\section{BACKGROUND}

According to the META Group, more than 70 percent of IT organizations are still perceived as cost centers rather than value centers. Managing the real and perceived business value of IT is thus very difficult.

With tight IT budgets, an effective and strategic way of managing IT investments becomes more critical for the success of IT organizations. Faced with this challenge, Intel IT developed the BVI methodology, a new approach to identify and evaluate potential benefits from making investments. BVI evaluates factors beyond the traditional cost framework. This approach helps identify the potential strategic value of an initiative and effectively communicate it to our executive management.

\section{WHAT IS THE BUSINESS VALUE INDEX?}

Some IT projects provide indirect benefits to a company, such as improving IT efficiency through optimized wide area network capacity while also delivering a new IT business capability to employees at a reduced cost. Other projects deliver direct benefits to a company by improving throughput, enhancing productivity, and increasing revenues. These projects deliver business value to the corporation but have no direct impact on the IT infrastructure. The Intel IT Business Value Index, shown in Figure 1, provides a way to conduct a relative comparison of these different IT initiatives to facilitate investment decisions that bring the most value to Intel and IT.

BVI is a composite index of factors that impact the value of an IT investment. It evaluates IT investments along three vectors: IT business value (that is, impact to Intel's business), impact to IT efficiency, and the financial attractiveness of an investment. All three factors use a predetermined set of defining criteria that includes customer need, business and technical risks, strategic fit, revenue potential, level of required investment, the innovation and learning generated, and other factors. Each factor's criteria are weighted according to the ongoing business strategy and 
business environment-changes in business strategy could change how criteria are weighted for different factors.

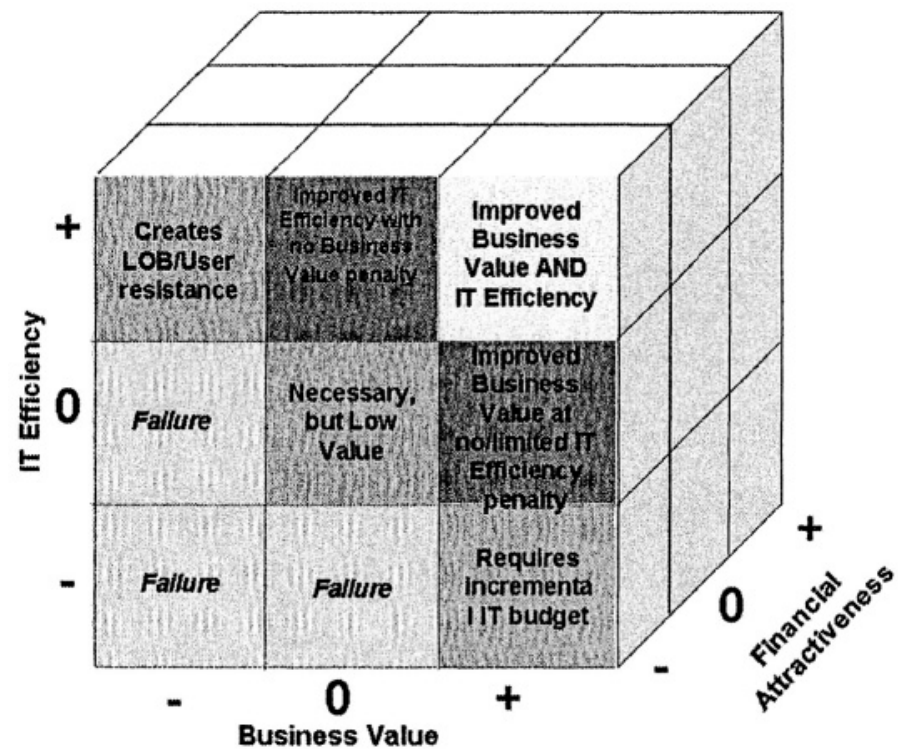

Figure 1. The Business Value Index

The predefined criteria include, but are not limited to:

- Customer need

- Business and technical risks

- Strategic fit

- Revenue potential

- Level of required investment

- Amount of innovation and learning generated

A crucial aspect of BVI is its ability to reveal the intangible benefits and strategic value of potential investments. It has a number of key characteristics that differentiate it from other prioritization methods. It provides a level comparison for multiple, strategic IT investment options. Weighted criteria support "what-if" analyses and rapid adjustments to changing business priorities. It tracks a potential investment's intangible benefits and strategic value, returning a series of decision points that depict the change in a project's relative value over a period of time.

BVI also highlights the most influential factors for assessing investments, indicates how each investment might add value to the company, and displays the results in a matrix that enhances comparative analysis of multiple investment opportunities. This approach supports options-based management of IT investments, helping us decide whether a project merits further funding. 
Using the BVI methodology, our management compares and contrasts investments, and then decides what investments align best with our business priorities.

\section{HOW IS THE BUSINESS VALUE INDEX USED?}

\subsection{Criteria and weightings}

Each vector in the BVI tool is comprised of a set of assessment criteria. Each criterion is weighted according to its importance in light of the business environment and current strategies. As project managers or program owners evaluate their proposed investments using the BVI tool, they score their project against these criteria on a scale of 0 to 3 , depending how the IT investment will likely perform against a range of values set for a particular assessment criteria. Table 1 shows a range of assessment criteria for a particular type of investment.

Table 1. Sample Assessment Criteria and Scoring

\begin{tabular}{lccccc}
\hline Criteria & Weight & 0 & 1 & 2 & 3 \\
\hline Customer pull/need & 4 & Low & Medium & High & Very high \\
\hline $\begin{array}{l}\text { Customer product cost } \\
\text { reduction }\end{array}$ & 3 & Increase & No impact & $\begin{array}{c}\text { Marginal } \\
\text { reduction }\end{array}$ & $\begin{array}{c}\text { Substantial } \\
\text { reduction }\end{array}$ \\
\hline $\begin{array}{l}\text { Business strategic fit } \\
\text { and impact }\end{array}$ & 3 & Low/NA & Medium & High & Very high \\
\hline $\begin{array}{l}\text { Customer performance } \\
\text { improvement }\end{array}$ & 3 & Decrease & $<5 \%$ & $>5 \%$ & $>10 \%$ \\
\hline
\end{tabular}

The products of the weighting and scoring of a particular investment's set of assessment criteria are summed to produce total scores for each of the three vectors-IT efficiency, financial attractiveness, and business value. After several projects have been scored, the Business Value Chart graphically depicts the three indices for each project. The position of a bubble relative to the horizontal and vertical axes shows each project's business value and IT efficiency values. The width of a bubble shows each project's financial attractiveness value. Figure 2, on the next page, depicts sample, evaluated projects.

The chart's graphical representation enables a quick assessment of investments relative to one another. The "win-win" circle shows that projects in the upper right corner of the chart have value both to IT and to Intel's business. Financial attractiveness is also important when evaluating the relative strength of various investments. 


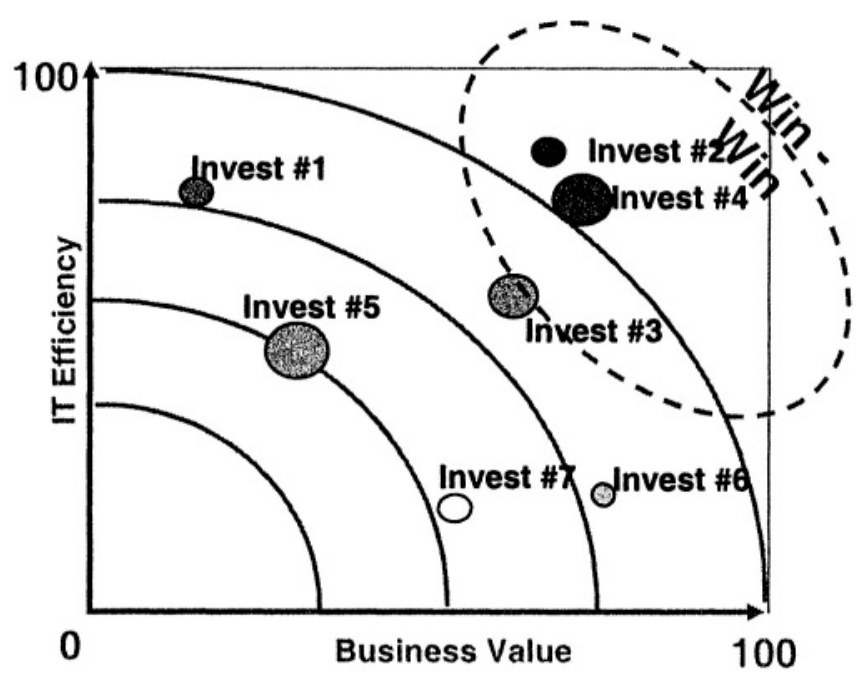

Figure 2. Sample Business Value Chart

Concentric circles identify projects with similar total BVI and IT efficiency index (ITI) scores. In Figure 2, for example, although investments 1 and 6 are on the same concentric circle, investment 1 is stronger in IEI, and investment 6 is stronger in BVI. The two investments have similar scores, but important differences exist. For that reason, business strategy and current priorities should guide the final prioritization of these projects, not solely the information on the chart.

Figure 3 shows a Project Comparison Matrix, which compares investments on a factor-by-factor basis. It shows the points accrued by each project in each factor minus the points lost. The matrix differentiates how the investments add value in different ways. Higher values in each section are shaded green or orange to visually depict the strengths and weaknesses of each project. The control values are shown at the bottom of the matrix.

This level of analysis - combined with knowledge of the group's current business strategy and environment-guides prioritization of investments. Differences in parts of the business or changes in business strategy can lead a team to adjust the weighting of different factors for analytical purposes. 


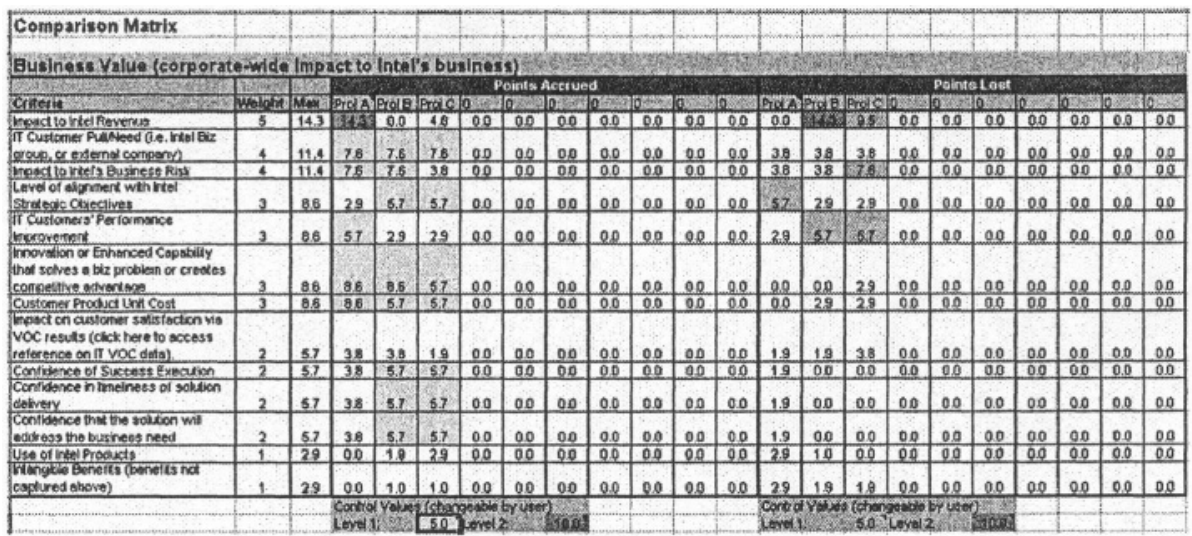

Figure 3. Sample Project Comparison Matrix

\subsection{Ongoing assessment process}

An ongoing Strategic Portfolio Management process continuously manages a team's investments, ensuring that investments are closely aligned with Intel IT's business strategies and priorities, and that IT resources are effectively utilized.

The Strategic Portfolio Management process is a closed-loop process using the BVI tool. The process starts with understanding IT's business strategies and then proposing projects to support those strategies. We access, prioritize, and rank proposals using BVI. Once the investment decisions are made, we commit resources based on the results of BVI and other qualitative data. The team builds monitors and indicators that track success at various stages of the project lifecycle.

We re-compute $\mathrm{BVI}$ on a regular basis to monitor the change in business value over time for the investments. Investment priorities can be adjusted based on the indicators and the BVI results. We track the actual results and benefits of the investment and measure them against the desired business results to provide feedback to the management process. This model's strength is that it enables continuous alignment between the IT investment portfolio and the dynamic business strategies and priorities. Actual results are measured against expectations set at the beginning of the investment. The model also enables data-driven decisions that may result in canceling projects or initiatives that fall short of expectations or no longer map to business priorities. 


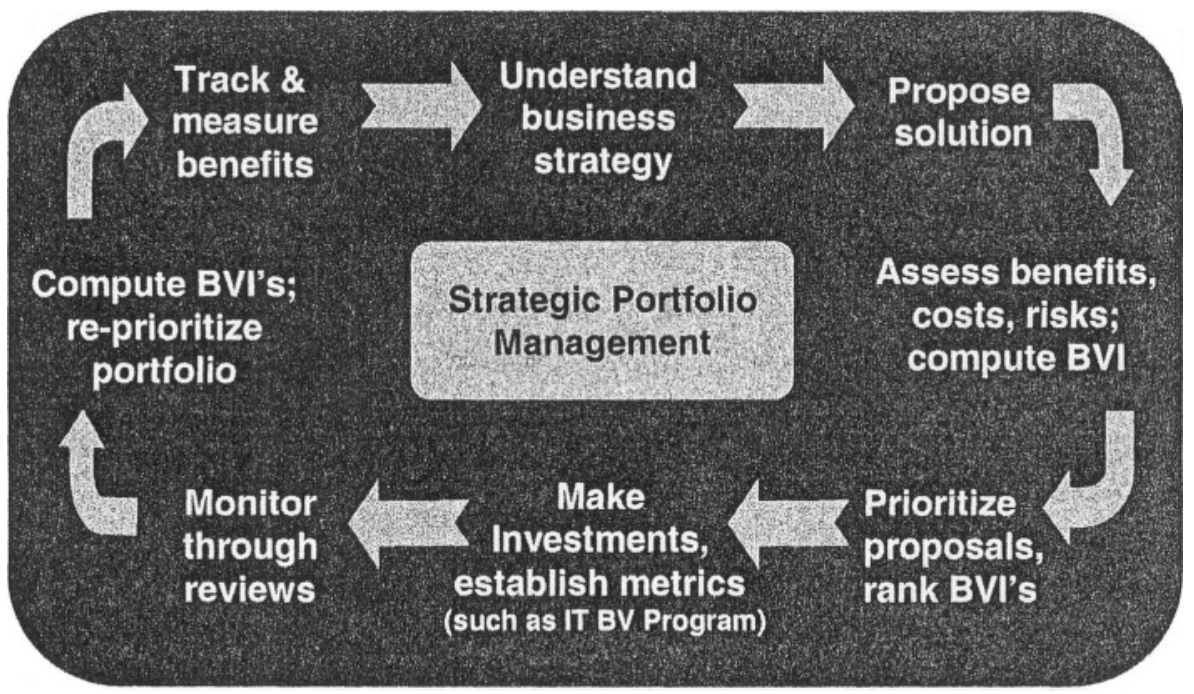

Figure 4. Ongoing portfolio management

\section{RESULTS}

BVI introduced a methodical, systematic approach to investment prioritization that supports data-driven decisions. Building on the BVI philosophy, we are implementing a strategic portfolio management framework that constitutes an integrated approach to investment decisionmaking and aims to identify and proactively manage a balanced investment portfolio mix. The framework encompasses common evaluation criteria (that is, BVI), investment categories and asset class definition, and visualization metrics.

BVI has assisted in decision-making activities over a wide variety of IT projects. For example, our Distributed Systems Management (DSM) team, People Systems group, IT Innovation organization, Customer Services group, and Global Infrastructure teams are using this tool to prioritize their project portfolios. We are using this approach to prioritize our IT Research and Development portfolio.

An example of a successful initiative prioritized through BVI is our Content Distribution System project, a peer-to-peer middleware services and knowledge management system. Using BVI, we prioritized the proof of concept (POC) for this project and gave it the go-ahead for development. Following the successful POC, this project has now emerged as a potential next-generation technology for IT.

Another example is our Electronic Content Distribution System (eCDS) project. We intercepted the development of eCDS before it was integrated 
into SKOOOL, a national educational portal in Ireland. The preliminary BVI evaluation proved accurate, resulting in a successful implementation. In parallel with SKOOOL, eCDS has also been integrated internally into an elearning site, and externally at the University of Reading, as part of a panEuropean, e-learning educational initiative.

\subsection{BVI adoption: Example}

The Telecom and Call Center Services (TCCS) group in our IT Global Infrastructure organization recently adopted BVI into their business process. TCCS is responsible for infrastructure projects funded by IT and for payper-view projects funded directly by IT customers. The group was looking for a method to help prioritize these diverse projects and make the right investment decisions. In light of the recent economic environment, prioritization became an even more important process in business management. TCCS turned to BVI as a potential tool to help solve their prioritization dilemmas.

A group composed of TCCS managers and project managers studied the BVI tool and started a pilot effort that evaluated whether BVI would fit their business requirements. The group selected a set of current and potential future projects for the pilot and used the BVI tool to conduct assessments.

As the pilot concluded, the group conducted a survey to gather feedback. The pilot team had found the tool valuable in helping them understand and assess the business impact of their projects and communicate that information more effectively to customers. The BVI tool also helped the team better prioritize their projects. The team commented that the time spent going through the process was well invested.

The group presented their findings to senior management, resulting in adoption of BVI as part of their ongoing business processes. Every project request is now associated with a BVI assessment before consideration for funding approval.

\section{CONCLUSION}

BVI provides a common language and framework for discussing IT investments, assessing business value and IT efficiency contribution based on common criteria, and prioritizing diverse investments based on environment and IT strategy. The BVI process enables continued and proactive alignment of the IT project portfolio with corporate and IT business strategies. 
Intel IT applies BVI to a variety of IT projects, which are then used by the ITBV metrics program. BVI creates an initial composite index to help gauge likely business benefits. The metrics program then looks more closely at the actual value delivered by a given project and assigns a real dollar value to the deliverables.

BVI is a decision support tool for IT investment decisions. It should not, however, be used as the only tool or factor in the decision making process. A thorough IT investment decision should be made based on the BVI results, the business drivers for different initiatives, the dependencies between projects (both within the group and between other groups), the impact to internal and external customers, the amount of investment, and other relevant business or environmental factors. 